\title{
Simulating wind effects on ponded membrane structures
}

\author{
N K. Narayanan ${ }^{\mathrm{a}, \mathrm{b}}$, R. Wüchner ${ }^{\mathrm{b}}$ and J. Degroote ${ }^{\mathrm{a}, \mathrm{c}}$ \\ ${ }^{a}$ Department of Electromechanical, Systems and Metal Engineering, Ghent University \\ Sint-Pietersnieuwstraat 41, 9000 Ghent \\ E-mail: navaneeth.narayanan@ugent.be \\ ${ }^{\mathrm{b}}$ Chair of Structural Analysis, Technical University of Munich, \\ Arcisstr. 21, 80333 Munich, Germany \\ ${ }^{\mathrm{c}}$ Flanders Make, Belgium
}

\begin{abstract}
In 2011, during the Pukkelpop festival held in Kiewit (Belgium), the sudden development of a storm resulted in strong wind gusts that led to extensive damage to the festival tents [1]. During this event, there was also heavy rainfall which caused ponding on the membrane structures. We believe one possible mechanism that resulted in the damage to the tents is the large vertical oscillation of the water mass with the membrane, excited by the fluctuating wind loads. This can easily be seen in the available video footages. The primary motivation of the current work is to simulate such a phenomenon. This requires unsteady fluid-structure interaction (FSI) simulation involving the wind, ponding water, and a membrane structure. In previous work [2], we developed methods for determining the initial condition of membrane structure with ponding for the unsteady FSI simulation, where the developed methods were used to calculate the static deformation of membrane structures under ponding loads.

In the present work, we propose a novel coupling strategy to solve such problems, where the wind, water, and membrane are simulated using three different solvers. The simulation of the wind is carried out by an OpenFOAM incompressible solver with large eddy simulation (wind solver), while for modeling the movement of water on the membrane an OpenFOAM volume of fluid solver (VoF) is used (water solver), and the structural solver with membrane elements in KratosMultiphysics is employed for modeling the membrane structure (structural solver).

The coupling strategy couples the structural solver in parallel with the wind and the water solver. This means that in the partitioned simulation, the structural solver receives tractions from the wind and water solvers, which are added and applied to the structure. On the other hand, the wind and the water solver receive displacement from the structural solver, used for updating the fluid-structure interface. The main assumption in this strategy is that the interaction between the water and the wind is not significant compared to the interaction between the two fluids and the structure. The results from this strategy are compared with the reference simulation, where the two fluids are simulated together in a VoF solver, which is coupled with the structural solver.
\end{abstract}

\section{REFERENCES}

[1] P. D. Meutter, L. Gerard, G. Smet, K. Hamid, R. Hamdi, D. Degrauwe and P. Termonia, "Predicting Small-Scale, Short-Lived Downbursts: Case Study with the NWP Limited-Area ALARO Model for the Pukkelpop Thunderstorm", Monthly Weather Review, American Meteorological Society, 143, 742-756 (2015)

[2] N.K. Narayanan, R. Wüchner and J. Degroote, "Monolithic and partitioned approaches to determine static deformation of membrane structures due to ponding", Computers \& Structures, 244, 106419 (2021). 